\title{
Aero-Optical Measurements in a Heated, Subsonic, Turbulent Boundary Layer
}

\author{
Jacob A. Cress ${ }^{*}$, Stanislav Gordeyev ${ }^{\dagger}$, and Eric J. Jumper ${ }^{\ddagger}$ \\ University of Notre Dame, Notre Dame, IN 46556, USA
}

\begin{abstract}
A scaling relationship for $\mathrm{OPD}_{\mathrm{rms}}$ that accounts for the fluctuating total temperature profile within a turbulent boundary is derived from the modified Crocco relation. Experimental data from heated, compressible boundary layers at six subsonic Mach numbers in two wind tunnel facilities are shown to be consistent with the theory. The results show that a temperature mismatch between the freestream and underlying wall has a significant impact on the overall optical aberration.
\end{abstract}

\section{Nomenclature}

\begin{tabular}{|c|c|c|c|}
\hline$c_{f}$ & $=$ skin friction coefficient & $\gamma$ & $=$ specific heat ratio \\
\hline$c_{p}$ & $=$ constant pressure specific heat & $\delta$ & $=$ boundary layer thickness \\
\hline$f$ & $=$ frequency & $\delta^{*}$ & $=$ displacement thickness \\
\hline$h$ & $=$ enthalpy & $\lambda$ & $=$ wavelength \\
\hline$I$ & $=$ instantaneous intensity on the optical axis & $\Lambda_{\rho}$ & $=$ density correlation length \\
\hline$I_{0}$ & $=$ diffraction limited intensity & $\Lambda_{u}$ & $=$ velocity correlation length \\
\hline$k$ & $=$ wavenumber $(2 \pi / \lambda)$ & $\rho$ & $=$ density \\
\hline$K_{G D}$ & $=$ Gladstone-Dale constant & $\sigma_{\rho^{\prime}}$ & $=$ mean-square density variance \\
\hline$M$ & $=$ Mach number & $\sigma_{\phi}$ & $=$ mean-square phase error \\
\hline OPD & $=$ optical path difference & $\varphi$ & $=$ deflection angle \\
\hline OPL & $=$ optical path length & Subscripts & \\
\hline$p$ & $=$ pressure & 0 & $=$ total property value \\
\hline $\operatorname{Pr}$ & $=$ Prandtl number & aw & $=$ adiabatic wall or recovery value \\
\hline$q$ & $=$ dynamic pressure & $c$ & $=$ convective value \\
\hline$r$ & $=$ recovery factor & norm & $=$ normalized \\
\hline$R$ & $=$ ideal gas constant & $p$ & $=$ peak value \\
\hline $\operatorname{Re}_{\theta}$ & $=$ Reynolds number & rms & $=$ root-mean-square \\
\hline St & $=$ Strouhal number & $S L$ & $=$ sea level conditions \\
\hline$S_{t}$ & $=$ Strehl ratio & $w$ & $=$ wall value \\
\hline$t$ & $=$ time & $\infty$ & $=$ freestream conditions \\
\hline$T$ & $=$ temperature & Superscripts & \\
\hline$u, U$ & $=$ streamwise velocity component & ' & $=$ fluctuating time or Reynolds avg \\
\hline$x$ & $=$ streamwise coordinate & - & $=$ mean time or Reynolds avg. \\
\hline$y$ & $=$ wall normal coordinate & $"$ & $=$ fluctuating Favre-averaging \\
\hline$\beta$ & $=$ elevation angle & $\sim$ & $=$ mean Favre-averaging \\
\hline
\end{tabular}

\section{Introduction}

$\mathrm{T}$ HE aero-optic problem is concerned with the study of wavefront aberrations caused by a variable index-of-refraction turbulent flow over an optical aperture, typically this distortion takes place within one to two aperture lengths of the aircraft. Free-shear layers, compressible boundary layers, shocks, and mixed density flows are potential sources for aero-optical aberrations. When a collimated laser beam with a planar (i.e. in-phase)

\footnotetext{
${ }^{*}$ Graduate Student, AME Department, B034 Hessert Laboratories, AIAA Student Member.

${ }^{\dagger}$ Research Assistant Professor, AME Department, 120 Hessert Laboratories, AIAA Member.

${ }^{\ddagger}$ Professor, AME Department, 110 Hessert Laboratories, AIAA Fellow.
} 
wavefront is projected through this variable index-of-refraction field, the emerging beam's wavefront will be distorted. These wavefront aberrations can have a severe negative effect on the performance of an optical-system, be it free-space communication, imaging, or directed-energy applications. ${ }^{1}$ Many different optical-system configurations have been envisioned: most employing an external turret that is exposed to the flow or an enclosed beam steering mirror with the aperture flush to the aircraft skin. The first configuration must contend with a complex flow field due to the presence of the turret in flow, while the latter configuration only has the aircraft's turbulent boundary layer over the aperture.

Turbulent boundary layers have been the subject of aero-optical research since the early 1950's with the first work being published by Liepmann in a Douglas Aircraft Company Technical Report. ${ }^{2}$ Liepmann measured the jitter angle of a thin light beam that was passed through the compressible boundary layers on the sides of high-speed wind tunnels as a means to quantify the crispness of Schlieren photographs. In 1956 Stine and Winovich performed a significant piece of work on light diffusion through turbulent boundary layers. ${ }^{3}$ This comprehensive report brought together the previous work in the field of optical propagation through variable index-of-refraction, turbulent flows. In this study, photometric measurements of the time-averaged radiation field at the focal plane of a receiving telescope were made in an attempt to verify Liepmann's formulation. A consequence of this work was the prospect of using the optical aberration measurements as a method of inferring turbulent scales. Sutton, in 1969, reported a theoretical formulation for the aberrating effect of turbulent boundary layers based upon statistical measures of the turbulence. ${ }^{4}$ The "linking equation" between turbulence quantities and optical phase variance is given as,

$$
\sigma_{\phi}^{2}=2 K_{G D}^{2} k^{2} \int_{0}^{L} \sigma_{\rho^{\prime}}^{2} \Lambda_{\rho}(y) d y,
$$

where $\sigma_{\phi}$ is the mean-square phase error, $K_{G D}$ is the Gladstone-Dale constant (which for air and visible wavelengths is approximately $\left.2.27 \times 10^{-4} \mathrm{~m}^{3} / \mathrm{kg}\right), k$ is the wavenumber $(2 \pi / \lambda), \sigma_{\rho^{\prime}}$ is the mean-square density variance, and $\Lambda_{\rho}$ is the density correlation length. Jumper and Fitzgerald ${ }^{1}$ showed that using the realization, first proposed by Malley et. $\mathrm{al}^{5}$, that aberrations caused by structures convecting in the turbulent flow themselves convect, Liepmann's and Sutton's formulations are equivalent.

Through the 1960's and 1970's, aero-optical studies of turbulent boundary layers intensified due to an interest in placing optical-systems on aircraft. In the 1970's Rose conducted an extensive experimental study of optical aberrations caused by a turbulent boundary layer. ${ }^{6}$ Using hotwire anemometry measurements taken at several wall normal locations within the turbulent boundary layer, Rose obtained the density fluctuations, $\rho^{\prime}$, and density correlation lengths, as a function of the wall normal location, $y$. It should be noted that in inferring $\rho^{\prime}$ from velocity measurements, Rose assumed that pressure fluctuations, $p^{\prime}$, were negligible. These measurements were then used to estimate the amount of aberration a wavefront would experience when propagated through the same boundary layer under the assumption of homogeneous turbulence. The on-average wavefront spatial aberrations, in the form of root-mean-square optical path difference, $\mathrm{OPD}_{\text {rms, }}$, were calculated using Sutton's linking equation, Eq. (1), which when expressed as $\mathrm{OPD}_{\mathrm{rms}}$ loses the $k^{2}$ term. Rose empirically found that $\mathrm{OPD}_{\mathrm{rms}}$ was proportional to dynamic pressure, $q$, and boundary layer thickness, $\delta$, such that $\mathrm{OPD}_{\mathrm{rms}} \sim q \delta$.

Rose's hotwire anemometry velocimeter results and optical aberration scaling relationship were complemented by Gilbert's 1982 interferometer measurements. ${ }^{7}$ Using a double pulse technique, Gilbert measured the difference in the aberrated wavefront from one pulse to the next. Gilbert found that the interferometry generally supported the velocity integral method of the linking equation as found by Rose. However, he concluded that the square of the $\mathrm{OPD}_{\mathrm{rms}}$ is linearly dependent on the dynamic pressure, $\mathrm{OPD}_{\mathrm{rms}}^{2} \sim q$. In 1994 Masson et al. ${ }^{8}$ revisited the Gilbert and Rose data and concluded that after removing systematic errors from Gilbert's data, $\operatorname{OPD}_{\mathrm{rms}} \sim\left(\rho M_{\infty}^{2}\right)^{1.16}$, where $M$ is the Mach number. Masson also found that there appeared to be a systemic difference between direct (i.e. interferometric) and indirect (i.e. the linking equation) wavefront error measurements, with the interferometric estimates consistently yielding higher estimates for $\mathrm{OPD}_{\mathrm{rms}}$ than the hotwire estimates, but he did not offer an explanation for why optical and velocimeter data did not agree.

By the 1990's, several experimental measurements of the $\mathrm{OPD}_{\mathrm{rms}}$ through a turbulent boundary layer had been performed, with estimates for the in-flight root-mean-square optical aberration being around $0.1 \mu \mathrm{m}$. The affect that wavefront aberrations have on an optical-system are quantified with the time-averaged Strehl ratio, $\overline{S_{t}}$, which is defined as $\overline{S_{t}}=I / I_{0}$, where $I$ is the instantaneous intensity on the optical axis and $I_{0}$ is the diffraction limited, distortion-free intensity value. The time-averaged Strehl ratio can be approximated using the $\mathrm{OPD}_{\mathrm{rms}}$ value and the "large-aperture approximation," 


$$
\left.\overline{S_{t}}=\exp \left[-\left(\frac{2 \pi \mathrm{OPD}}{\lambda}\right)^{\mathrm{rms}}\right)^{2}\right]
$$

where $\lambda$ is the laser wavelength. ${ }^{9}$ Lasers being used on airborne platforms in the 1960's and 1970's primarily used $\mathrm{CO}_{2}$ lasers that have a lasing wavelength of $10.6 \mu \mathrm{m}$. Using the large-aperture approximation, the time-averaged Strehl ratio through a turbulent boundary layer at this wavelength is $99.65 \%$. As a result, turbulent boundary layers were initially thought to have little or no impact of the optical-system performance. ${ }^{1}$ However, as Eq. (2) shows, for a fixed value of $\mathrm{OPD}_{\mathrm{rms}}$, decreasing the laser wavelength will have the effect of decreasing the time-averaged Strehl ratio. Optical-systems being considered today use near infrared and visible wavelengths $(\sim 1 \mu \mathrm{m})$, which have an order of magnitude shorter wavelength than the $\mathrm{CO}_{2}$ laser, decreasing the time-averaged Strehl ratio to $67.38 \%$. Therefore, the turbulent boundary layer that did not previously factor into the optical-system design because of the small impact on far-field beam intensity now has a discernable time-averaged influence.

Based on this fact, turbulent boundary layer investigations picked up in the 1990's. In 1992, Malley et al. ${ }^{5}$, introduced a pioneering new instrument to measure wavefront aberrations using a small-aperture beam which would be deflected by the turbulent flow. Gordeyev et al ${ }^{10}$ advanced the described instrument by adding a closely spaced beam in the streamwise flow direction in order to extract convective velocity using Taylor's frozen flow assumption. The resulting Malley probe gives accurate, highly time-resolved information (exceeding $100 \mathrm{kHz}$ ) on the deflection of the small-aperture beam at a single location. Beam deflection angle, $\varphi$, is related to streamwise OPD by,

$$
\begin{aligned}
& \operatorname{OPL}(t)=-U_{c} \int_{0}^{t} \varphi(t) d t, \\
& \operatorname{OPD}(t)=\operatorname{OPL}(t)-\overline{\operatorname{OPL}(t)},
\end{aligned}
$$

where OPL is the optical path length, defined as the integral of the index-of-refraction along the beam propagation length, and $U_{c}$ is the convective velocity of the optically active flow structures. The reader is directed to [10] for further information and a discussion of the Malley probe setup, operation, and data analysis. Wyckham and Smits ${ }^{17}$ used a two-dimensional Shack-Hartmann sensor to study aero-optical properties of subsonic and supersonic boundary layers and developed a scaling law for these aberrations as a function of the freestream Mach number, the local skin friction coefficient, $c_{f}$, and the ratio of the freestream static temperature, $T_{\infty}$, to the wall temperature, $T_{w}$,

$$
\mathrm{OPD}_{\mathrm{rms}}=0.96 K_{G D} \delta M_{\infty}^{2} \sqrt{c_{f}}\left(\frac{T_{w}}{T_{\infty}}+1\right)^{-3 / 2} \text {. }
$$

Aero-optical studies on turbulent boundary layers have been an ongoing topic of research at the University of Notre Dame since the early 2000's. Gordeyev et al. ${ }^{11}$ performed optical measurements at several Mach numbers and for various boundary layer thicknesses, from which they concluded that the $\mathrm{OPD}_{\mathrm{rms}}$ scaled with density, displacement thickness, $\delta^{*}$, and Mach number squared, $\mathrm{OPD}_{\mathrm{rms}} \sim \rho_{\infty} / \rho_{S L} \delta^{*} M_{\infty}{ }^{2}$, where the initial constant of proportionality was found to be $2.4 \times 10^{-5}$. This scaling is consistent with the scaling of Wyckham and Smits ${ }^{17}$ in the limit of moderate subsonic speeds and adiabatic wall conditions. Bucker et al.$^{12}$ verified this scaling using a simple vortical structure model, though his model over-estimated the experimental $\mathrm{OPD}_{\mathrm{rms}}$ values by a factor of 4 . In 2007 , Wittich et al. ${ }^{13}$ made improvements to the experimental apparatus and employed new data analysis techniques to refine the constant of proportionality to $(1.6 \pm 0.4) \times 10^{-5}$. The new analysis technique also showed that the optically active structures in the flow (with an approximate displacement-thickness-based Strouhal number of 0.1) convected with a velocity of approximately 0.87 of the freestream, suggesting that the optical activity was in the outer region of the boundary layer, with the mean structure's size of about the boundary layer thickness. Wittich's study also verified that when passing through two statistically independent boundary layers, the contribution to the overall root-mean-square optical aberration through both boundary layers was equivalent to the square root of the sum of the two boundary layers squared. ${ }^{13}$ The $\mathrm{OPD}_{\mathrm{rms}}$ relationship between the double boundary layer, $\mathrm{DBL}$, and two single boundary layers, SBL, is therefore, 


$$
\mathrm{OPD}_{\mathrm{rms}}^{\mathrm{DBL}}=\sqrt{\left(\mathrm{OPD}_{\mathrm{rms}}^{\mathrm{SBL}, 1}\right)^{2}+\left(\mathrm{OPD}_{\mathrm{rms}}^{\mathrm{SBL}, 2}\right)^{2}}
$$

Cress et al. ${ }^{14}$ adapted the proposed scaling relationship to include a dependence on elevation angle, $\beta$, and updated the coefficient of proportionality, giving the current scaling relationship as,

$$
\mathrm{OPD}_{\mathrm{rms}}=(1.7 \pm 0.2) \times 10^{-5} \frac{\delta^{*}}{\sin (\beta)} \frac{\rho_{\infty}}{\rho_{S L}} M_{\infty}^{2} .
$$

Further, the connective velocity was found to be approximately 0.83 of the freestream, slightly slower than that measured by Wittich et al. ${ }^{13}$

For nearly as long as turbulent boundary layers have been studied, those with heat transfer at the wall have been the subject of experimental and computational research, especially applied to supersonic and hypersonic boundary layers. ${ }^{15-21}$ Smits ${ }^{15}$ and the review article by Spina ${ }^{16}$ are excellent compilations of information on heat transfer in the compressible, turbulent boundary layer. A significant advancement in the field came from Morkovin's 1962 presentation of the Strong Reynolds Analogy (SRA) ${ }^{15-17}$, which presumes that $p^{\prime}$ is negligible. From this analogy between the Reynolds-averaged form of the energy and momentum equations, a relationship between the fluctuating static temperature and fluctuation velocity is achieved, such that,

$$
\frac{T^{\prime}}{\bar{T}}=-(\gamma-1) M^{2} \frac{u^{\prime}}{\bar{U}}
$$

where $T$ is the static temperature (in absolute scale), $\gamma$ is the specific heat ratio, and $u, U$ is the local streamwise, $x$, velocity component; primes indicate fluctuating quantities and overbars indicate mean quantities. Note that Eq. (7) is strictly valid only when the Prandtl number, $\mathrm{Pr}$, is unity. The SRA has been empirically verified for Mach numbers up to three. ${ }^{16}$ However, the SRA, consistent with the assumption that $p^{\prime}$ is approximately zero, neglects fluctuations in the total temperature in a boundary layer. For turbulent boundary layers without heat transfer at the wall, this assumption does not introduce significant error. ${ }^{15-17}$ But for non-adiabatic wall conditions, experimental and computational studies have shown that fluctuations in the total temperature can be as large as $60 \%$ of the fluctuating static temperature, thus the fluctuating total temperature cannot be ignored. ${ }^{18,20}$

The research conducted at the University of Notre Dame has not previously accounted for the potential temperature mismatch between the underlying wall and freestream, so the current study investigates the impact that heat transfer at the underlying wall of a subsonic, compressible, turbulent boundary layer has on optical aberrations. A simple statistical model for the $\mathrm{OPD}_{\mathrm{rms}}$ as a function of subsonic Mach number and moderate temperature difference is derived using the modified Crocco relation in Section II. The relationship is compared against experimental data from a heated wall experiment in Section IV, which is followed by discussion and conclusions in Section V.

\section{Statistical Model}

The SRA presumes that the total temperature fluctuations are zero; however, Walz and van Driest proposed a form of the enthalpy equation such that $\tilde{h}=h(\tilde{u})$, where fluctuations in the total temperature are not ignored. ${ }^{15}$ Carrying out the analysis similar to [15], the following relationships for mean and fluctuating static temperature are found,

$$
\begin{gathered}
\frac{\tilde{T}}{T_{\infty}}=\frac{\tilde{T}_{w}}{T_{\infty}}+\frac{\tilde{T}_{a w}-\tilde{T}_{w}}{T_{\infty}}\left(\frac{\tilde{u}}{U_{\infty}}\right)-r \frac{(\gamma-1)}{2} M_{\infty}^{2}\left(\frac{\tilde{u}}{U_{\infty}}\right)^{2}, \\
\frac{T^{\prime \prime}}{T_{\infty}}=-\frac{\tilde{T}_{a w}-\tilde{T}_{w}}{T_{\infty}}\left(\frac{u^{\prime \prime}}{U_{\infty}}\right)-r \frac{\tilde{u} u^{\prime \prime}}{c_{p} T_{\infty}}
\end{gathered}
$$

where $\tilde{T}, \tilde{T}_{\infty}, \tilde{T}_{w}$, and $\tilde{T}_{a w}$ are the Favre-averaged static, freestream, wall, and adiabatic or recovery temperatures, respectively, $r$ is the recovery factor, $c_{p}$ is the constant pressure specific heat, $\tilde{u}$ is the Favre-averaged mean 
velocity, and $u^{\prime \prime}$ is the Favre-averaged fluctuating velocity. Equation (8a) is known as the modified Crocco relation or the Walz equation. The recovery factor is defined,

$$
r=\frac{\tilde{T}_{a w}-\tilde{T}_{\infty}}{\tilde{T}_{0_{\infty}}-\tilde{T}_{\infty}}
$$

The difference between Reynolds and Favre-averaging has been shown to be less than $1.5 \%$ for Mach numbers less than 3, thus they can be interchanged for this subsonic and low supersonic analysis. ${ }^{15}$ Letting $\Delta T=T_{w}-T_{a w}$, and replacing fluctuating values with root-mean-square values, the following expression for $T_{\text {rms }}$ can be found,

$$
\left(\frac{T_{\mathrm{rms}}}{T_{\infty}}\right)^{2}=\left(\frac{u_{\mathrm{rms}}}{U_{\infty}}\right)^{2} \cdot\left[\left(\frac{\Delta T}{T_{\infty}}\right)^{2}+2 r(\gamma-1) M_{\infty}^{2}\left(\frac{\Delta T}{T_{\infty}}\right) \frac{U}{U_{\infty}}+\left(r(\gamma-1) M_{\infty}^{2} \frac{U}{U_{\infty}}\right)^{2}\right],
$$

where $U$ is the mean local streamwise velocity.

From the ideal gas law, $p=\rho R T$, and assuming that pressure fluctuations in the boundary layer are much smaller than density and temperature fluctuations, ${ }^{15-17}$ (this assumption will be further discussed in Section V) it follows that,

$$
\frac{\rho^{\prime}}{\rho_{\infty}}=-\frac{T^{\prime}}{T_{\infty}} \longrightarrow\left(\frac{\rho_{\mathrm{rms}}}{\rho_{\infty}}\right)^{2}=\left(\frac{T_{\mathrm{rms}}}{T_{\infty}}\right)^{2}
$$

Substituting Eq. (10) into Eq. (11) gives the following relationship for $\left(\rho_{\mathrm{rms}}\right)^{2}$ in terms of the velocity profile in the wall normal direction and $\Delta T$,

$$
\left(\frac{\rho_{\mathrm{rms}}}{\rho_{\infty}}\right)^{2}=\left(\frac{u_{\mathrm{rms}}}{U_{\infty}}\right)^{2} \cdot\left[\left(\frac{\Delta T}{T_{\infty}}\right)^{2}+2 r(\gamma-1) M_{\infty}^{2}\left(\frac{\Delta T}{T_{\infty}}\right) \frac{U}{U_{\infty}}+\left(r(\gamma-1) M_{\infty}^{2} \frac{U}{U_{\infty}}\right)^{2}\right] .
$$

Equation (1) expressed in terms of $\mathrm{OPD}_{\mathrm{rms}}$ and with Eq. (12) substituted in for the square of fluctuating density variance, results in the following relationship,

$$
\mathrm{OPD}_{\mathrm{rms}}=B_{0} \delta^{*} \frac{\rho_{\infty}}{\rho_{S L}}\left[B_{1} M^{4}+B_{2} \frac{\Delta T}{T_{\infty}} M^{2}+B_{3}\left(\frac{\Delta T}{T_{\infty}}\right)^{2}\right]^{1 / 2}
$$

where,

$$
\begin{aligned}
& B_{0}=\sqrt{2} K_{G D} \rho_{S L}(\gamma-1), \\
& B_{1}=\int_{0}^{\infty}\left[r^{2}\left(\frac{U(y) u_{\mathrm{rms}}(y)}{U_{\infty}^{2}}\right)^{2} \frac{\Lambda_{\rho}(y)}{\delta^{*}}\right] d\left(\frac{y}{\delta^{*}}\right), \\
& B_{2}=\int_{0}^{\infty}\left[\frac{2 r}{(\gamma-1)} \frac{U(y)}{U_{\infty}}\left(\frac{u_{\mathrm{rms}}(y)}{U_{\infty}}\right)^{2} \frac{\Lambda_{\rho}(y)}{\delta^{*}}\right] d\left(\frac{y}{\delta^{*}}\right), \\
& B_{3}=\int_{0}^{\infty}\left[\left(\frac{u_{\mathrm{rms}}(y)}{U_{\infty}(\gamma-1)}\right)^{2} \frac{\Lambda_{\rho}(y)}{\delta^{*}}\right] d\left(\frac{y}{\delta^{*}}\right) .
\end{aligned}
$$

Rewritten with $A=B_{0} B_{1}{ }^{1 / 2}, C_{1}=B_{2} / B_{1}$, and $C_{2}=B_{3} / B_{1}$, Eq. (13) becomes, 


$$
\mathrm{OPD}_{\mathrm{rms}}=A \delta^{*} \frac{\rho_{\infty}}{\rho_{S L}}\left[M^{4}+C_{1} \frac{\Delta T}{T_{\infty}} M^{2}+C_{2}\left(\frac{\Delta T}{T_{\infty}}\right)^{2}\right]^{1 / 2}
$$

Note that Eq. (14) reduces to the previous scaling relation given by Eq. (6) (neglecting the elevation dependence on $\delta^{*}$ ) if the difference between the wall temperature and adiabatic wall temperature is zero. Equation (14) can be rearranged in the following manner,

$$
\begin{gathered}
\mathrm{OPD}_{\mathrm{rms}}=A \delta^{*} \frac{\rho_{\infty}}{\rho_{S L}}\left[\left(M^{2}+D_{1} \frac{\Delta T}{T_{\infty}}\right)^{2}+D_{2}\left(\frac{\Delta T}{T_{\infty}}\right)^{2}\right]^{1 / 2}, \\
\mathrm{OPD}_{\mathrm{rms}}=A \delta^{*} \frac{\rho_{\infty}}{\rho_{S L}}\left(M^{2}+D_{1} \frac{\Delta T}{T_{\infty}}\right)\left[1+\frac{D_{2}}{2}\left(\frac{\Delta T / T_{\infty}}{M^{2}+D_{1} \Delta T / T_{\infty}}\right)^{2}+\text { H.O.T. }\right],
\end{gathered}
$$

where $D_{1}=C_{1} / 2$ and $D_{2}=C_{2}-\left(C_{1} / 2\right)^{2}$. If the last term in the square brackets of Eq. (15b) can be shown to be much less than 1 , then,

$$
\mathrm{OPD}_{\mathrm{rms}}=A \delta^{*} \frac{\rho_{\infty}}{\rho_{S L}}\left(M^{2}+D_{1} \frac{\Delta T}{T_{\infty}}\right)
$$

becomes the scaling relationship for $\mathrm{OPD}_{\mathrm{rms}}$ as a function of Mach number and temperature difference. This assumption will be discussed and shown valid over the range of tested Mach numbers and $\Delta T$ values in Sections IV.A and V. The $\mathrm{OPD}_{\mathrm{rms}}$ scaling relationship given by Eq. (16) will be compared to experimental data obtained in a heated, compressible, subsonic turbulent boundary layer.

\section{Experimental Setup}

Double boundary layer measurements were conducted in two wind tunnel facilities at the Hessert Laboratories for Aerospace Research at the University of Notre Dame. Five Mach numbers, 0.2, 0.3, 0.4, 0.5, and 0.6, were tested in the transonic facility and one Mach number, 0.12 , was tested in the low speed wind tunnel. The transonic wind tunnel facility, shown schematically in Figure 1, is an indraft tunnel with an inlet contract ratio of 150:1, followed by a boundary layer development section, measurement section, and diffuser. The development and measurement sections have a constant cross-sectional area measuring $10.1 \mathrm{~cm}$ by $9.9 \mathrm{~cm}$. The measurement section was located $156 \mathrm{~cm}$ downstream from the beginning of the development section. The value of the freestream velocity in the measurement section was obtained with total and static pressure ports. A variable speed vacuum pump was used to precisely lower the pressure in a plenum to which the diffuser was attached, achieving the desired

\section{SIDE VIEW}

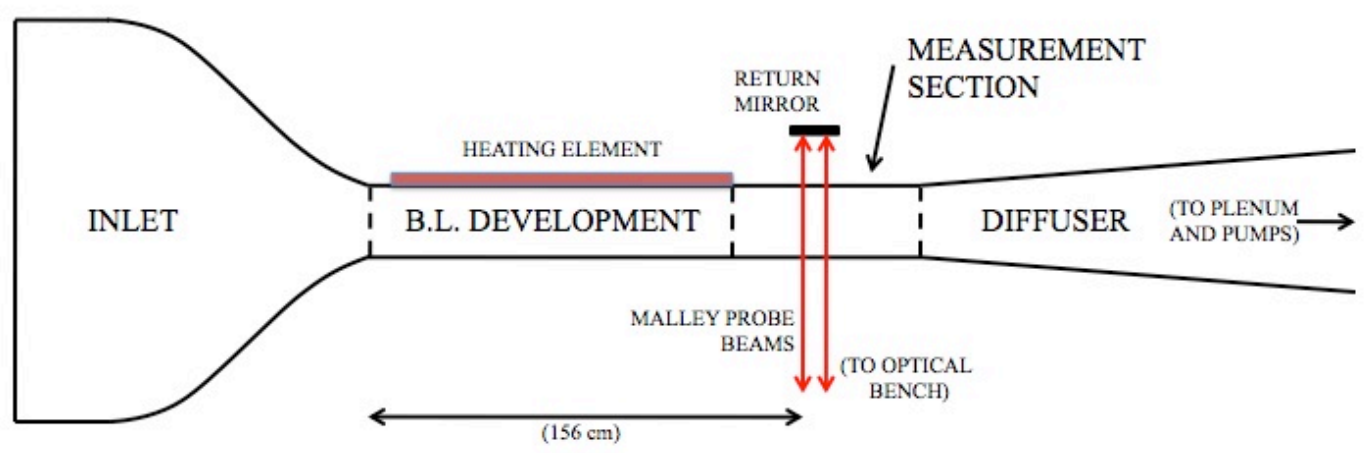

Figure 1. Schematic of the Notre Dame transonic turbulent boundary layer test facility configured for heated boundary layer measurements. 
flow velocity. The indraft low speed wind tunnel facility has a test section with a constant cross-sectional area of $2 \mathrm{ft}$ by $2 \mathrm{ft}$. The distance from the beginning of the boundary layer development section to the measurement location was approximately $150 \mathrm{~cm}$. The freestream velocity was measured with a pitot-static probe. The tunnel was powered with a single stage fan rotating at $55 \mathrm{~Hz}$.

In both facilities, one wall of the boundary layer development section was replaced with an aluminum plate that had flexible, electric resistive coil heaters thermally epoxied to the outside surface and insulated, see Figure 1 . The temperature of the heaters were controlled with a PID circuit with the temperature input coming from a thermal tab embedded flush to the inside wall which measured the wall surface temperature. The thickness of the aluminum plate was chosen to be relatively thick to ensure a uniform wall surface temperature.

The boundary layer mean and rms velocity profiles in each facility were quantified with hotwire anemometry measurements at several streamwise locations. The transonic facility boundary layer was measured with a freestream Mach number of 0.5 and the low speed wind tunnel was measured at Mach 0.12.

Optical measurements were performed in each facility using a Malley probe. The beams were propagated in the wall normal direction through the heated and the unheated boundary layer on each wall at the measurement location for the six Mach numbers stated above. Several wall temperatures were tested at each Mach number; it should be noted that due to the fixed output power of the heaters, the maximum achievable wall temperature was dependent on the flow speed with a greater difference between freestream and wall temperature achieved in the transonic tunnel at Mach $0.2(\Delta T \sim 28 \mathrm{~K})$ than at Mach $0.6(\Delta T \sim 15 \mathrm{~K})$. The low speed tunnel had a slightly different heater configuration that allowed a maximum temperature difference of $10 \mathrm{~K}$.

\section{Results}

\section{A. Velocity Measurements}

Hotwire anemometry velocity measurements were performed at Mach 0.12 in the low-speed tunnel and at Mach 0.5 in the transonic tunnel. The normalized mean and rms profiles are shown in Figure 2. The mean velocity profiles for the two facilities show excellent agreement for $y / \delta^{*}$ greater than 1 , below this value the low speed tunnel decreases in velocity faster than the transonic tunnel with the slowest measurable point in the Mach 0.12 data being approximately $0.3 U_{\infty}$ while the lowest measured transonic tunnel velocity was about $0.5 U_{\infty}$. The two facility rms velocities, however, differ over a larger region of the boundary layer. While each are of the same magnitude, the fluctuating velocity at Mach 0.5 is greater than the Mach 0.12 profile between 2 and $7 \delta^{*}$.

Gilbert ${ }^{7}$ provides values for the streamwise velocity correlation length, $\Lambda_{u}$, as a function of the wall normal direction, see Figure 3. From the mean and rms velocity profile results and the Gilbert streamwise velocity correlation lengths, which are directly related to density correlation lengths, it is possible to evaluate the $B$ coefficients given in Eq. (13) and then solve for the coefficients $A, C_{1}$, and $C_{2}$. A recovery factor of 0.89 was assumed. For the low speed, $M=0.12$, using the measured velocity profile, $A$ was calculated to be $1.45 \times 10^{-5}$, and
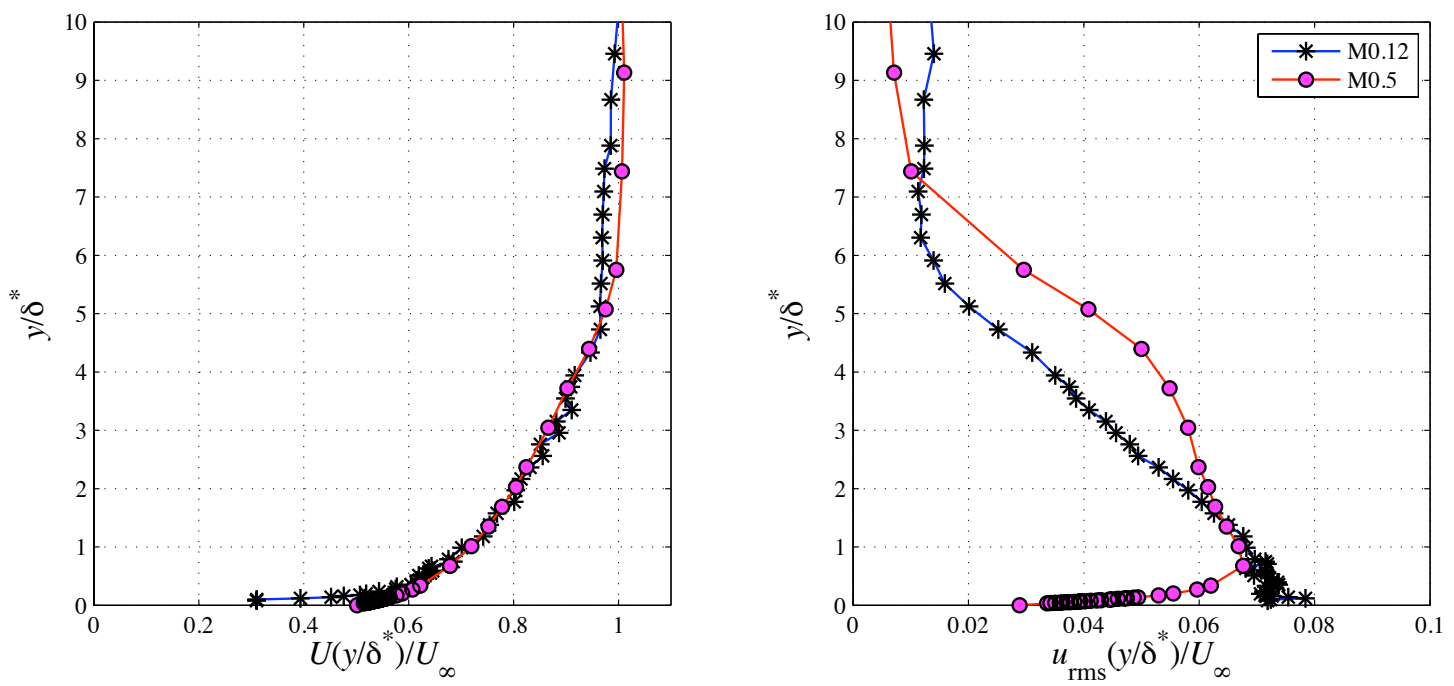

Figure 2. Normalized mean (left) and rms (right) velocity profiles at Mach 0.5 in the transonic wind tunnel facility and at Mach 0.12 in the low speed tunnel facility.

American Institute of Aeronautics and Astronautics 


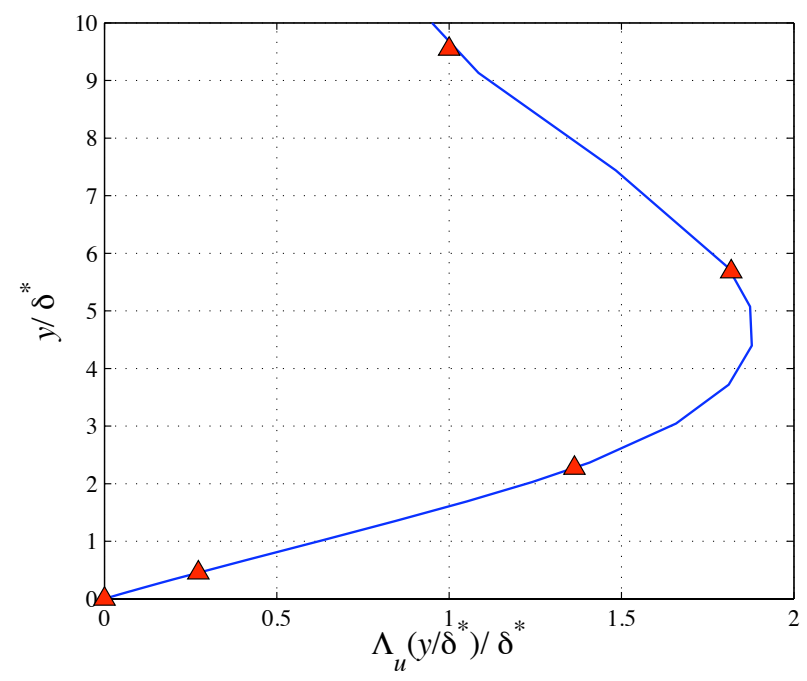

Figure 3. Streamwise velocity correlation lengths, $\Lambda_{u}$, normalized by the displacement thickness, $\delta^{*}$ as a function of the wall normal direction. [Gilbert 1982]

$C_{1}$ and $C_{2}$ were found to be 6.59 and 10.99 , respectively. At Mach $0.5, A, C_{1}$, and $C_{2}$ were determined to be $1.89 \times 10^{-5}, 6.38$, and 10.28 , respectively. The calculated values of $A$ are consistent with the experimentally measured $^{14}$ constant of $1.7 \times 10^{-5}$. For $M=0.12$, these values of $C_{1}$ and $C_{2}$ give $D_{1}$ and $D_{2}$ constant values of 3.29 and 0.15 , respectively; at the higher $M=0.5$, the values for these constants were calculated to be $D_{1}=3.19$ and $D_{2}=0.1$. To verify that Eq. (15) can be simplified into Eq. (16), for the tested Mach number and temperature ranges, the second term in Eq. (15b) must be shown to be much less than 1. Inspection of that term at two Mach numbers of 0.12 and 0.5 and with the calculated values of $D_{1}$ and $D_{2}$, shows the value of that term to be less than 0.007 for temperature differences as large as $2 T_{\infty}$; suggesting that Eq. (16) is a valid simplification for the present study.

\section{B. Deflection Angle Spectra}

From Eq. (3) it follows that the deflection angle spectrum, $\hat{\varphi}(f)$, is proportional to $\mathrm{OPD}_{\text {rms }}$ divided by $U_{\infty}$,

$$
\hat{\varphi}(f) \propto \frac{1}{U_{\infty}} \mathrm{OPD}_{\mathrm{rms}}
$$

where $f$ is frequency. Therefore, using Eq. (5), the deflection angle spectrum for a single boundary layer is,

$$
\hat{\varphi}(f)_{\mathrm{SBL}, \text { heated }}=\sqrt{\left(\hat{\varphi}(f)_{\mathrm{DBL}, \text { heated }}\right)^{2}-\frac{1}{2}\left(\hat{\varphi}(f)_{\mathrm{DBL}, \text { unheated }}\right)^{2}} \text {. }
$$

Figure 4 (left) shows the spectrum amplitude scaled to a single boundary layer for five Mach numbers and near zero difference between the wall and adiabatic wall temperature, $\Delta T \sim 0$. The narrow band spikes present in the spectrum data are at a fixed frequency for all Mach numbers and are related to electronic noise in the data acquisition system.

Using Eq. (17) and the $\mathrm{OPD}_{\mathrm{rms}}$ scaling derived in Eq. (16), the deflection angle spectrum can be expressed as,

$$
\hat{\varphi}(f)=\frac{\delta^{*}}{U_{\infty}} \frac{\rho_{\infty}}{\rho_{S L}}\left(M^{2}+D_{1} \frac{\Delta T}{T_{\infty}}\right) \cdot \hat{\varphi}_{\text {norm }}(\mathrm{St})
$$

where $\hat{\varphi}_{\text {norm }}(\mathrm{St})$ is introduced as a normalized deflection angle spectrum and $\mathrm{St}$ is the Strouhal number, $\mathrm{St}=f \delta^{*} / U_{\infty}$. The right plot in Figure 4 shows the normalized spectrum for five Mach numbers at $\Delta T \sim 0$. The scaling collapses the data fairly well, though there are discrepancies. The slope of the roll-off steadily becomes more negative from the Mach 0.2 curve (blue) to the Mach 0.6 curve (cyan). This change in slope is the result of the zero level noise being sufficiently large relative to the optical signal to artificially boost the high frequencies at the lower Mach 

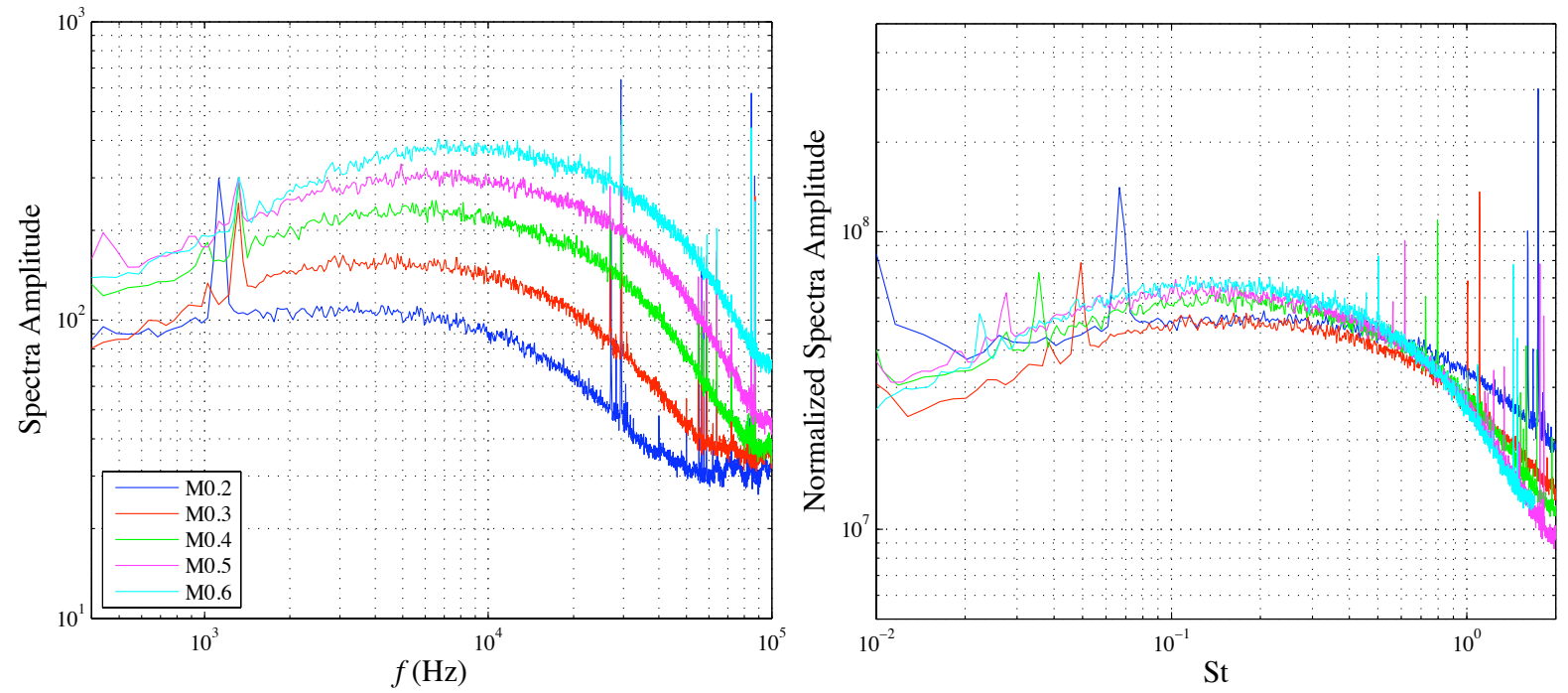

Figure 4. (left) Spectra amplitude for the turbulent boundary layer at five Mach numbers and $\Delta T \sim 0$. (right) Normalized spectra amplitude for the same five Mach number and $\Delta T \sim 0$.

numbers; by Mach 0.5 the slope has reached a constant value. Also, in the peak region, St $\sim 0.1$, the two lower Mach numbers, 0.2 and 0.3 , are slightly lower than the other cases. This result appears to be a real feature of the turbulence and becomes more apparent as the temperature difference is increased.

To determine the value of the $D_{1}$ constant in Eq. (16), the peak of the spectrum amplitude corresponding to the maximum value near $\mathrm{St}=0.1$, was investigated as a function of temperature difference. The left plot in Figure 5 shows the peak values of the spectra amplitudes for the six tested Mach numbers and numerous temperature differences. Writing Eq. (19) in terms of the peak spectrum amplitude and solving for $D_{1} \Delta T / T_{\infty}$ gives,

$$
\frac{U_{\infty}}{\delta^{*} \rho_{\infty} / \rho_{S L}}\left(\frac{\hat{\varphi}_{p, \text { heated }}-\hat{\varphi}_{p, \text { unheated }}}{\hat{\varphi}_{p, \text { norm }}}\right)=D_{1} \frac{\Delta T}{T_{\infty}} .
$$

The normalized peak values of the spectra amplitude are plotted versus $\Delta T / T_{\infty}$ in Figure 5 (right). Of interest is that the value of $D_{1}$ increases with Mach number below 0.3. The values of $D_{1}$ are plotted versus the Reynolds number based on momentum thickness, $\operatorname{Re}_{\theta}$, in Figure 6 . It can be seen that the value of $D_{1}$ increases until approximately a
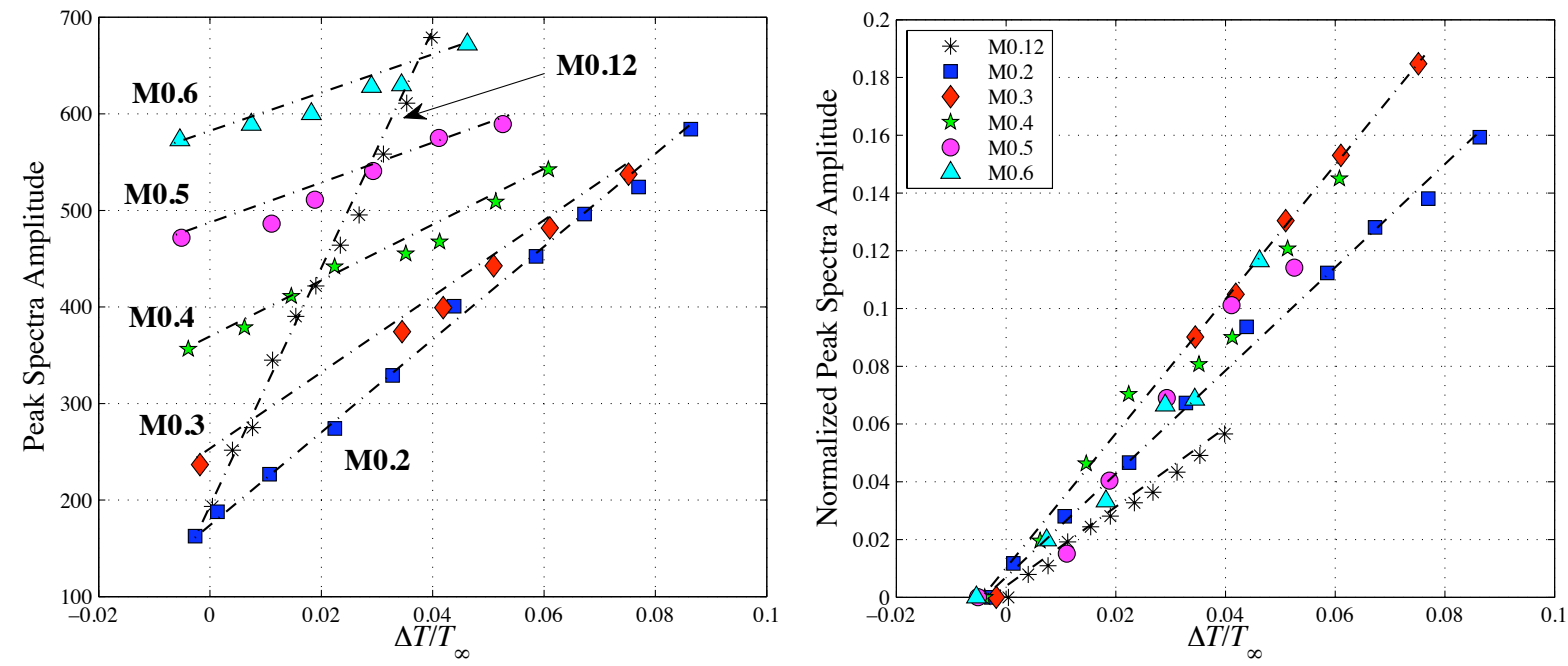

Figure 5. (left) Peak values from the spectra amplitude at six Mach numbers and a range of temperature differences. (right) Normalized peak values of the spectra amplitude, Eq. (20), plotted versus $\Delta T / T_{\infty}$. 


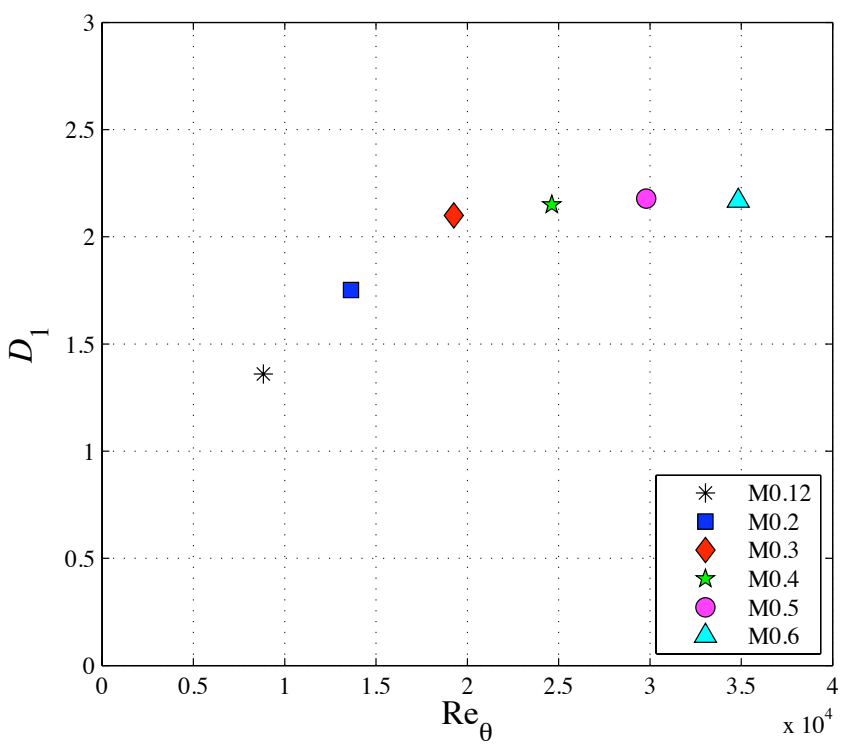

Figure 6. Equation (16) $D_{1}$ "constant" plotted versus Reynolds number based on momentum thickness.

$\mathrm{Re}_{\theta}$ of 20,000 ; for larger $\mathrm{Re}_{\theta}$ values $D_{1}$ settles on a constant value of about 2.15. The experimental values of $D_{1}$ are approximately 1.5 times less than the values calculated from the mean and rms velocity profiles and correlation lengths in Section IV.A. Additionally, the calculated values from the velocity profiles do not show the Reynolds number dependence seen in the experimental data at lower $\operatorname{Re}_{\theta}$ values. The simplification from Eq. (15) to Eq. (16) has been empirically shown valid for low subsonic flow with moderate temperature differences; therefore another effect must be the source for the $D_{1}$ dependence on Reynolds number.

Shown in Figure 7 (left), are the normalized spectra amplitudes at Mach 0.4 for two temperature difference extremes, $\Delta T \sim 0$ and $19.2 \mathrm{~K}$, where the temperature difference term has been neglected in the normalization (i.e. $D_{1}=0$ ). The right plot in Figure 7 shows the normalized spectra amplitudes for the same conditions but now including the temperature difference in the normalization with $D_{1}$ as found by Eq. (20) and Figure 5 (right). The proposed normalization for temperature dependence from Eq. (19) shows an excellent collapse of the experimental data. As previously discussed, the two cases show some disagreement in the slope of the roll-off at higher frequencies. However, the location of the peak spectrum value near $\mathrm{St} \sim 0.1$ is not affected with the change in temperature difference, and moreover the shape of the spectrum in general was not altered, but rather it was just
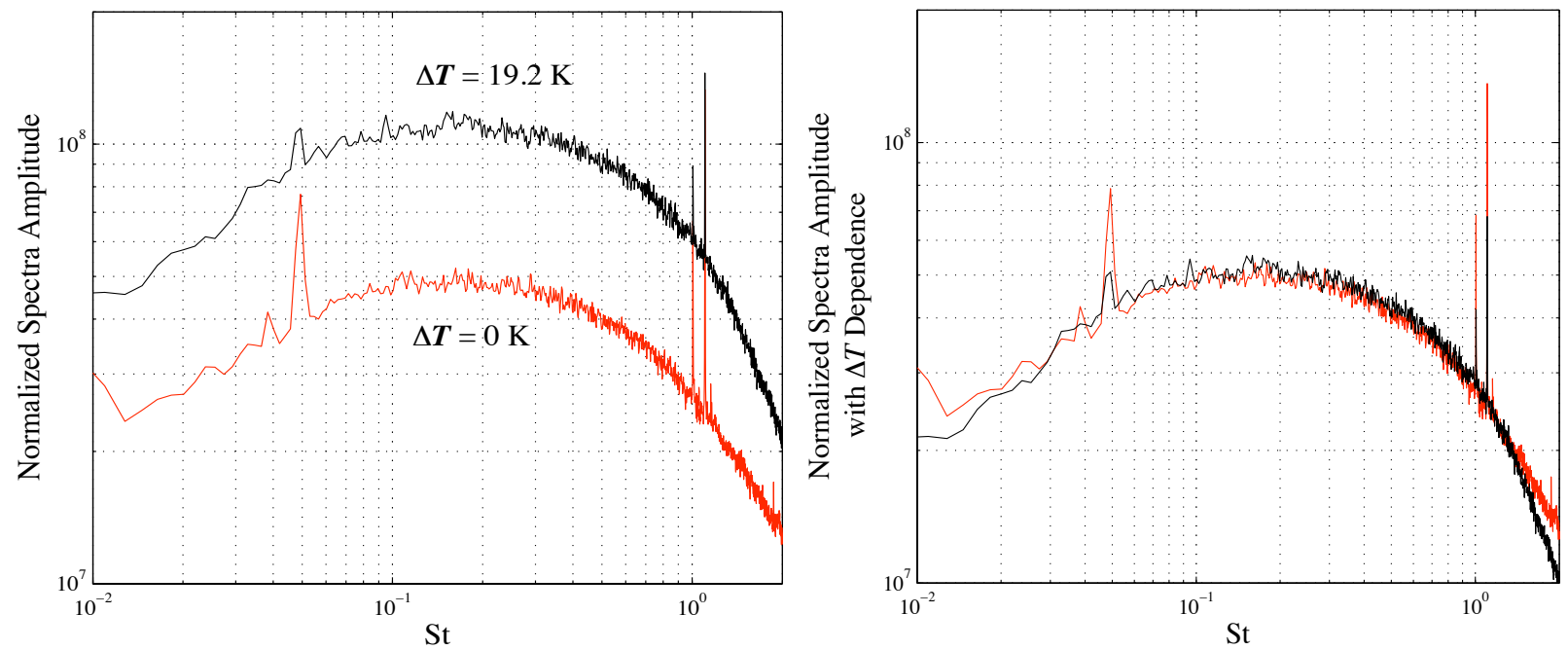

Figure 7. (left) Normalized spectra amplitude, Eq. (19), neglecting the temperature dependence (i.e. $D_{1}=0$ ), for the Mach 0.4 turbulent boundary layer at two $\Delta T$ extremes, 0 and $19.2 \mathrm{~K}$. (right) Normalized spectra amplitude with temperature dependence included at the same conditions.

10

American Institute of Aeronautics and Astronautics 
linearly shifted vertically with $\Delta T$. This suggests that the effect of temperature difference, at least in the regime studied, did not significantly change the overall statistical properties of the boundary layer structures, but only amplified the fluctuating density values within the structures. Also, that the spectrum was linearly amplified across a large range of St values, suggests that the temperature difference equally affected a broad scale of structure sizes.

The complete set of normalized spectra at Mach 0.2, 0.3, 0.4, 0.5, and 0.6, at the temperature difference extremes, $\Delta T \sim 0$ and $\Delta T_{\max }$, are shown in Figure 8. For each of the Mach numbers, the linear temperature scaling successfully collapses the two temperature differences to a single curve. However, as was seen in the normalization for $\Delta T \sim 0$ in Figure 4 (right), the two lower Mach numbers, 0.2 and 0.3 , are clearly separated from the higher Mach number cases. As the Mach number increases, the spectra appear to better collapse with the proposed scaling. As a consequence of the data failing to collapse to a universal curve, it is possible that the Mach number dependence, not accounted for in the proposed scaling in Eq. (19), may be due to the boundary layer being in transition between Mach 0.3 and 0.4. In addition to the spectra amplitudes not definitively collapsing, the location of the peak value of the spectra, which occurs near St $\sim 0.1$, does slightly increase as the Mach number decreases.

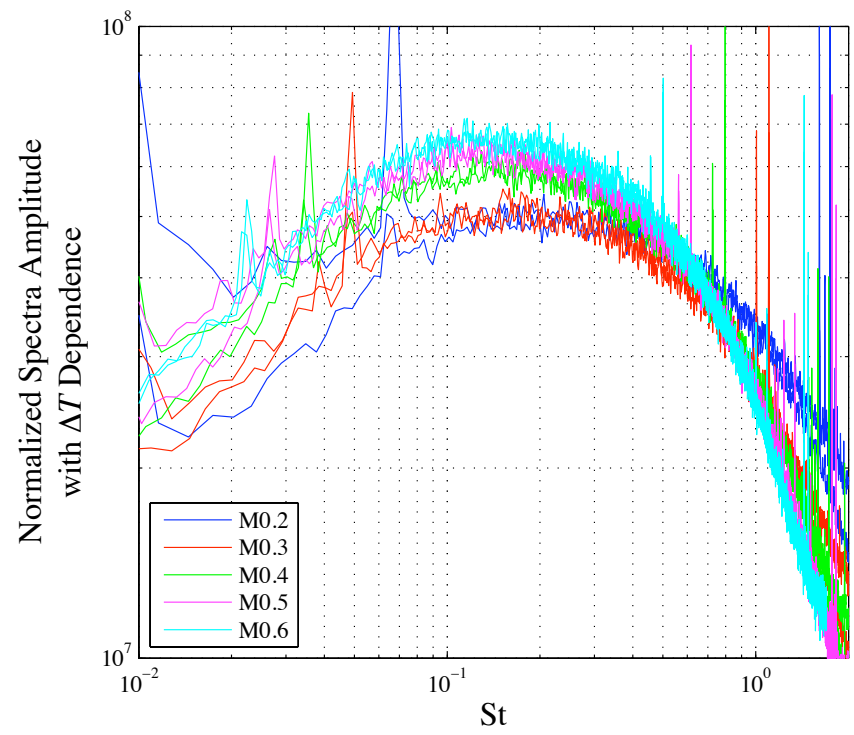

Figure 8. Normalized spectra amplitude, Eq. (19), for Mach 0.2, 0.3, 0.4, 0.5, and 0.6 at the temperature difference extremes, $\Delta T \sim 0$ and $\Delta T_{\max }$.

\section{C. $O P D_{\text {rms }}$ Scaling}

The value of $\mathrm{OPD}_{\mathrm{rms}}$ was found by taking the root-mean-square of the calculated OPD time series, found by multiplying the integral of the deflection angle time history by the convection velocity, as given by Eq. (3). Prior to integration, the deflection angle time series was high-pass filtered at $300 \mathrm{~Hz}$ to remove low frequency vibration contamination. $\mathrm{OPD}_{\mathrm{rms}}$ is plotted versus the scaling relation given by Eq. (16) for five Mach numbers and several temperature differences in Figure 9. It is evident from this figure that the scaling relation successfully collapses the $\mathrm{OPD}_{\mathrm{rms}}$ values over a wide range of subsonic Mach numbers and $\Delta T$ values. The slope, $A$, was found to be $1.7 \times 10^{-5}$, which is consistent with the previously reported value of $(1.7 \pm 0.2) \times 10^{-5}$ by Cress et al. ${ }^{14}$ These results show that a temperature mismatch between the wall and the freestream of $28 \mathrm{~K}$ at Mach 0.2 can more than double the value of $\mathrm{OPD}_{\mathrm{rms}}$ that an optical-system would experience. Thus, the effect of varying total temperature in the wall normal direction cannot be ignored in the turbulent boundary layer.

From the simple statistical model, Eq. (16), and experimental data in Figure 9, it is clear that $\mathrm{OPD}_{\mathrm{rms}}$ increases with increasing wall temperature, $T_{w}$. The model proposed by Wyckham and Smits ${ }^{17}$ in fact gives the opposite trend, since from their model, Eq. (4), $\mathrm{OPD}_{\mathrm{rms}} \sim\left(T_{w} / T_{\infty}+1\right)^{-3 / 2}$, predicts that $\mathrm{OPD}_{\mathrm{rms}}$ decreases with increasing wall temperature. 


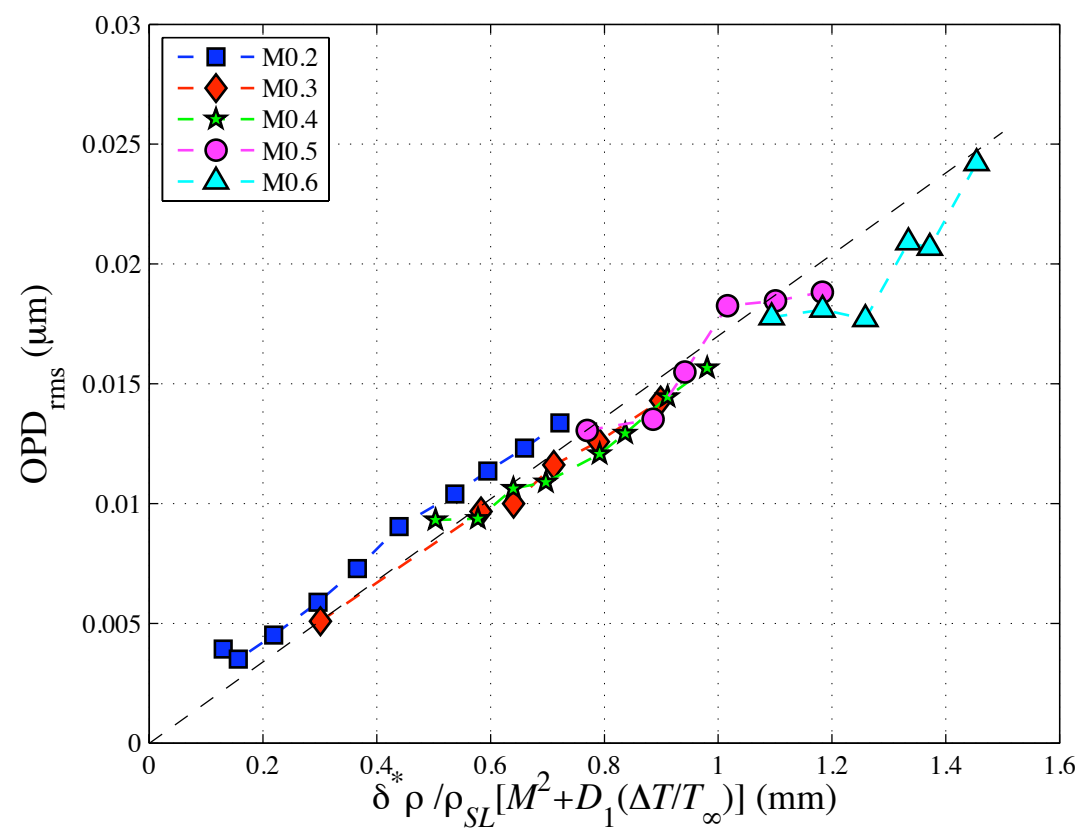

Figure 9. $O$ PD $_{\text {rms }}$ versus the scaling relationship given by Eq. (16) and the $D_{1}$ constants found by the spectra analysis for five Mach numbers and several temperature differences. The constant of proportionality, $A$, was found to be $1.7 \times 10^{-5}$.

\section{Conclusions and Discussion}

The work presented in this paper shows that heat addition at the wall in a subsonic, compressible turbulent boundary layer has an important impact on the optical aberration of a transmitted wavefront. Further, a simple model for the $\mathrm{OPD}_{\mathrm{rms}}$ for subsonic boundary layers was derived from the modified Crocco relation, which assumes that the streamwise pressure gradient is zero. This relation does not assume a constant total temperature across the boundary layer. The derived scaling relationship for $\mathrm{OPD}_{\mathrm{rms}}$, incorporating the boundary layer thickness, elevation angle, altitude, Mach number, and temperature dependencies is given as,

$$
\mathrm{OPD}_{\mathrm{rms}}=(1.7 \pm 0.2) \times 10^{-5} \frac{\delta^{*}}{\sin (\beta)} \frac{\rho_{\infty}}{\rho_{S L}}\left(M^{2}+D_{1} \frac{\Delta T}{T_{\infty}}\right),
$$

where the $D_{1}$ constant is thought to be a function of $\operatorname{Re}_{\theta}$, see Figure 6. The values found experimentally for $A$ and $D_{1}$, were consistent with values calculated using the mean and rms velocity profiles and the velocity correlation lengths as reported by Gilbert. ${ }^{7}$

The validity of Eq. (21) for positive $\Delta T$ values has been empirically shown with a set of heated wall experiments at several Mach numbers and in two wind tunnel facilities. The effect on $\mathrm{OPD}_{\mathrm{rms}}$ due to temperature dependencies in a heated wall have been shown to be successfully scaled by application of the modified Crocco relation and the linking equation between velocity and optical fluctuations. From these results, some thoughts on the effect of cooling the wall have been considered. The non-simplified form of the $\mathrm{OPD}_{\mathrm{rms}}$ scaling, Eq. (14), takes a quadratic form with respect to the temperature difference. The location of the minimum value of the $\mathrm{OPD}_{\mathrm{rms}}$ is found at $\Delta T / T_{\infty}=-\frac{1}{2} C_{1} M^{2} / C_{2}$. At Mach 0.12 , this corresponds to a value of $-0.004\left(\Delta T=-1.3^{\circ} \mathrm{C}\right.$ assuming $\left.T_{\infty}=300 \mathrm{~K}\right)$, while at Mach 0.5, the minimum location moves to -0.078 (or $\Delta T=-23.3^{\circ} \mathrm{C}$ ). The deviation between the linear scaling, Eq. (16), and the true quadratic Eq. (14) becomes important as this minimum $\Delta T / T_{\infty}$ location is approached from positive temperature differences and passed into large negative differences. Therefore, for small negative $\Delta T$ values relative to the Mach number squared, the proposed linear scaling with temperature remains valid. However, for large negative $\Delta T$ quantities, the quadratic form of the $\mathrm{OPD}_{\mathrm{rms}}$, Eq. (14), is required. An implication from this extension into cooled walls is that some limited amount of heat extraction at the wall will lead to a decrease in the 
optical aberration over an aperture, where the $\mathrm{OPD}_{\mathrm{rms}}$ based on the $\Delta T / T_{\infty}$ value at the minimum of Eq. (14) is $0.1 A \delta^{*} \rho_{\infty} / \rho_{S L} M_{\infty}^{2}$, or $10 \%$ of the value at zero $\Delta T$. Since most aero-optical applications would experience a set of flight conditions where the aircraft skin is at an elevated temperature relative to the freestream temperature, decreasing the skin temperature could greatly improve the optical environment.

From the spectra results, it is apparent that for the range of $\Delta T$ values tested, the moderate temperature contamination from the heated wall simply amplifies the density fluctuations in the boundary layer with little or no effect on the statistics of the boundary layer structures size and shape. Further, the energy in the amplitude spectrum varied linearly with temperature difference. Both results are consistent with the theory derived in Eq. (16). This result suggests that low speed flows with very low levels of optical aberrations can be made optically active by introducing a temperature mismatch between the wall and freestream; therefore, optical aberrations that were unobservable at $\Delta T=0$ could be measured with common optical wavefront instruments without distorting the optically relevant structures, simply by heating the wall.

Further work is necessary to complete the temperature contamination picture. Larger positive temperature differences need to be investigated to determine at what temperature buoyancy and gravity effects become a factor in the movement, organization and optical characteristics of boundary layer structures. The value of $D_{1}$ needs to be further investigated to determine its functional dependence on $\mathrm{Re}_{\theta}$ and Mach number; also, the presumption that $p^{\prime}$ equals to zero which led to the value of $D_{1}$ needs to be revisited. Finally, cooled wall experiments need to be performed to investigate whether the derived $\mathrm{OPD}_{\mathrm{rms}}$ continues to decrease to a minimum value as the temperature is decreased as predicted by Eq. (14).

\section{Acknowledgments}

This work is supported in part by the Air Force Office of Scientific Research, Grant number FA9550-09-1-0449. The U.S. Government is authorized to reproduce and distribute reprints for governmental purposes notwithstanding any copyright notation thereon.

\section{References}

${ }^{1}$ Jumper, E.J. and Fitzgerald, E.J., "Recent advances in aero-optics," Progress in Aerospace Sciences, Vol. 37, No. 3, 2001, pp. 299-339.

${ }^{2}$ Liepmann, H.W., "Deflection and Diffusion of a Light Ray Passing Through a Boundary Layer," Report SM-14397, Douglas Aircraft Company, Santa Monica Division, California, 16 May 1952.

${ }^{3}$ Stine, H.A. and Winovich, W., "Light Diffusion Through High-Speed Turbulent Boundary Layers," NACA Research Memorandum, NACARMA-56B21, Washington, 25 May 1956.

${ }^{4}$ Sutton, G.W., "Effect of Turbulent Fluctuations in an Optically Active Fluid Medium," AIAA Journal, Vol. 7, No. 9, September 1969, pp. 1737-1743.

${ }^{5}$ Malley, M.M, Sutton, G.W., and Kincheloe, N., "Beam-jitter measurements of turbulent aero-optical path differences," Applied Optics, Vol. 31, No. 22, 1 August 1992, pp. 4440-4443.

${ }^{6}$ Rose, W.C., "Measurements of Aerodynamic Parameters Affecting Optical Performance," Air Force Weapons Laboratory Final Report, AFWRL-TR-78-191, May 1979.

${ }^{7}$ Gilbert, K.G., "KC-135 Aero-Optical Boundary-Layer/Shear-Layer Experiments," Aero-Optical Phenomena, Eds. K.G. Gilbert and L.J. Otten, Vol. 80, Progress in Astronautics and Aeronautics, AIAA, New York, 1982, pp. $306-324$.

${ }^{8}$ Masson, B., Wissler, J., and McMackin, L., "Aero-Optical Study of a NC-135 Fuselage Boundary Layer," AIAA Paper 94-0277, January 1994.

${ }^{9}$ Smith, W.J., Modern Optical Engineering: The Design of Optical Systems, McGraw-Hill, New York, 1966, Chapter 3, pp. 49-71.

${ }^{10}$ Gordeyev, S., Hayden, T.E., and Jumper, E.J., "Aero-Optical and Flow Measurements Over a Flat-Windowed Turret," AIAA Journal, Vol. 45, No. 2, February 2007, pp. 347-357

${ }^{11}$ Gordeyev, S., Jumper, E.J., Ng, T.T., and Cain, A.B., "Aero-Optical Characteristics of Compressible, Subsonic Turbulent Boundary Layers," 34 $4^{\text {th }}$ AIAA Plasmadynamics and Lasers Conference, AIAA-2003-3603, Orlando, FL, $23-26$ June 2003.

${ }^{12}$ Buckner, A., Gordeyev, S., and Jumper, E.J., "Optical Aberrations Caused by Transonic Attached Boundary Layers: Underlying Flow Structure," $43^{\text {rd }}$ AIAA Aerospace Sciences Meeting and Exhibit, AIAA-2005-0752, Reno, NV, 10-13 January 2005.

${ }^{13}$ Wittich, D.J., Gordeyev, S., and Jumper, E.J., "Revised Scaling of Optical Distortions Caused by Compressible, Subsonic Turbulent Boundary Layers," $38^{\text {th }}$ AIAA Plasmadynamics and Lasers Conference, AIAA-2007-4009, Miami, FL, 25-28-June 2007.

${ }^{14}$ Cress, J.A., Gordeyev, S., Post, M.L., and Jumper, E.J., “Aero-Optical Measurements in a Turbulent, Subsonic Boundary Layer at Different Elevation Angles," $39^{\text {th }}$ AIAA Plasmadynamics and Lasers Conference, AIAA-2008-4214, Seattle, WA, 23-26 June 2008. 
${ }^{15}$ Smits, A.J. and Dussauge, J.P., Turbulent Shear Layers in Supersonic Flow, American Institute of Physics, Woodbury, New York, 1996, Chapter 5.

${ }^{16}$ Spina, E.F., Smits, A.J., and Robinson, S.K., "The Physics of Supersonic Turbulent Boundary Layers," Annual Review of Fluid Mechanics, Vol. 26, 1994, pp. 287-319.

${ }^{17}$ Wyckham, C.M. and Smits, A.J., "Aero-Optic Distortion in Transonic and Hypersonic Turbulent Boundary Layers," AIAA Journal, Vol. 47, No. 9, September 2009, pp. 2158-2168.

${ }^{18}$ Morinishi, Y., Tamano, S., and Nakabayaski, K., "Direct numerical simulation of compressible turbulent channel flow between adiabatic and isothermal walls," Journal of Fluid Mechanics, Vol. 502, 2004, pp. 273-308.

${ }^{19}$ Martín, M.P., "Mach Number and Wall Temperature Effects on Turbulent Boundary Layers," Conference on Turbulence and Interactions, Porquerolles, France, 29 May-2 June 2006.

${ }^{20}$ Rani, S.L., Smith, C.E., and Nix, A.C., "Boundary-Layer Equation-Based Wall Model for Large-Eddy Simulation of Turbulent Flows with Wall Heat Transfer," Numerical Heat Transfer, Part B, Vol. 55, 2009, pp. 91-115.

${ }^{21}$ Fulachier, L. and Dumas, R., "Spectral analogy between temperature and velocity fluctuations in a turbulent boundary layer,” Journal of Fluid Mechanics, Vol. 77, Part 2, 1976, pp. 257-277. 